\title{
Existence and approximate controllability of Hilfer fractional evolution equations with almost sectorial operators
}

\author{
Pallavi Bedi' ${ }^{1}$ Anoop Kumar ${ }^{1}$, Thabet Abdeljawad ${ }^{2,3,4^{*}} \mathbb{D}$, Zareen A. Khan ${ }^{5}$ and Aziz Khan²
}

\section{"Correspondence:}

tabdeljawad@psu.edu.sa

${ }^{2}$ Department of Mathematics and

General Sciences, Prince Sultan

University, 66833, 11586 Riyadh,

Saudi Arabia

${ }^{3}$ Department of Medical Research, China Medical University, 40402,

Taichung, Taiwan

Full list of author information is

available at the end of the article

\section{Springer}

\begin{abstract}
In this article, we are concerned with the existence of mild solutions and approximate controllability of Hilfer fractional evolution equations with almost sectorial operators and nonlocal conditions. The existence results are obtained by first defining Green's function and approximate controllability by specifying a suitable control function. These results are established with the help of Schauder's fixed point theorem and theory of almost sectorial operators in a Banach space. An example is also presented for the demonstration of obtained results.
\end{abstract}

MSC: Primary 26A33; secondary 34K37; 93B05

Keywords: Hilfer fractional derivative; Almost sectorial operator; Approximate controllability; Nonlocal conditions

\section{Introduction}

In current times, the rising interest of researchers in fractional calculus reflects the popularity of this branch $[1-3,5-13,15,19,30-32]$. Differential equations of fractional order are widely applicable in the areas of physics, chemistry, electromagnetics, and mechanics. Fractional differential equations (FDEs) have been used widely in the identification of different physical systems, in control theory, in simulating viscoelastic materials, and in the modeling of different complex phenomena [22-29, 39, 42]. The concept of exact controllability and approximate controllability of FDEs is an active field of investigation because of its major applications in physical sciences. Under some admissible control inputs, exact controllability steers the system to arbitrary final state, while approximate controllability steers the system to the small neighborhood of arbitrary final state. In the published works, there are numerous articles focussing on the exact or approximate controllability of systems represented by FDEs, neutral FDEs, FDEs with impulsive inclusions, and FDEs with delay functions $[4,18,40,44]$.

In particular, approximate controllability of Hilfer FDEs under different conditions has been discussed widely. Mahmudov et al. [36] investigated the exact controllability of Hilfer FDEs in a Hilbert space under the assumption that a linear system of the given equation is approximate controllable. In 2017, Yang et al. [41] discussed the approximate controllabil-

(c) The Author(s) 2020. This article is licensed under a Creative Commons Attribution 4.0 International License, which permits use sharing, adaptation, distribution and reproduction in any medium or format, as long as you give appropriate credit to the original author(s) and the source, provide a link to the Creative Commons licence, and indicate if changes were made. The images or other third party material in this article are included in the article's Creative Commons licence, unless indicated otherwise in a credit line to the material. If material is not included in the article's Creative Commons licence and your intended use is not permitted by statutory regulation or exceeds the permitted use, you will need to obtain permission directly from the copyright holder. To view a copy of this licence, visit http://creativecommons.org/licenses/by/4.0/. 
ity of Hilfer FDEs with nonlocal conditions in a Banach space with the help of semigroup theory, fixed point techniques, and multivalued analysis. Later on, Debbouche et al. [14] and Du et al. [17] studied the approximate controllability of Hilfer FDEs and semilinear Hilfer FDEs with impulsive control inclusions in Banach spaces, respectively. In 2018, Lv and Yang [34] investigated the approximate controllability of neutral Hilfer FDEs by applying the techniques of stochastic analysis theory and semigroup operator theory in a Hilbert space. In recent works, Lv and Yang [35], with the help of Banach contraction principle, discussed the approximate controllability for a class of Hilfer FDEs of order $1<\alpha<2$ and type $0 \leq \beta \leq 1$. It is noted that, in almost all the problems discussed above, a linear operator generates the strongly continuous semigroup of bounded linear operators.

In [37] Periago and Straub formulated theory to analyze almost sectorial operators. Here authors also mentioned the suitable assumptions required to establish the existence of mild solutions and classical solutions of FDEs with almost sectorial operator. There are numerous works focussing on the existence of mild solutions and analytical solutions of fractional evolution equations with almost sectorial operators [16, 33, 43]. Recently, Jaiswal et al. [21] proved the existence of mild solutions of Hilfer FDEs with almost sectorial operators. We found that in the available literature, approximate controllability of Hilfer FDEs with almost sectorial operators has not been discussed yet. Thus, motivated by the above-discussed works, we consider here the following system of Hilfer FDEs for investigating proposed results:

$$
\left\{\begin{array}{l}
{ }^{H} \mathcal{D}_{0^{+}}^{v, \mu} v(\mathfrak{t})=\mathcal{A} v(\mathfrak{t})+\Upsilon w(\mathfrak{t})+\Psi\left(\mathfrak{t}, v(\mathfrak{t}), \int_{0}^{\mathfrak{t}} \mathfrak{q}(\mathfrak{t}, \mathfrak{s}) \varphi(\mathfrak{t}, \mathfrak{s}, v(\mathfrak{s})) d \mathfrak{s}\right), \quad \mathfrak{t} \in \mathfrak{J} \\
\mathcal{I}_{0^{+}}^{(1-v)(1-\mu)} v(0)=\sum_{k=1}^{\mathfrak{m}} c_{k} v\left(\mathfrak{t}_{k}\right),
\end{array}\right.
$$

where ${ }^{H} \mathcal{D}_{0^{+}}^{v, \mu}$ is the Hilfer fractional derivative of order $0<v<1$ and type $0 \leq \mu \leq 1$. $\mathcal{A}$ : $D(\mathcal{A}) \subset \mathcal{V} \longrightarrow \mathcal{V}$ is an almost sectorial operator in Banach space $\mathcal{V}$ and $\mathfrak{J}=[0,6], 6>0$. The control function $w(\mathfrak{t})$ takes value in $\mathcal{L}^{2}(\mathfrak{J}, \mathcal{W}) . \mathcal{L}^{2}(\mathfrak{J}, \mathcal{W})$ denotes the space of admissible control functions for Banach space $\mathcal{W} . \Upsilon$ is a bounded linear operator from $\mathcal{W}$ into $\mathcal{V}$. $\Psi: \mathfrak{J} \times \mathcal{V} \times \mathcal{V} \longrightarrow \mathcal{V}$ is a continuous linear mapping, $0<\mathfrak{t}_{1}<\mathfrak{t}_{2}<\cdots<\mathfrak{t}_{\mathfrak{m}}<6, \mathfrak{m} \in \mathbb{N}$, and $c_{\kappa}$ are real numbers such that $c_{\kappa} \neq 0$. The characteristics functions $\mathfrak{q}(\mathfrak{t}, \mathfrak{s}): \Delta \longrightarrow \mathbb{R}$ and $\varphi: \Delta \times \mathcal{V} \longrightarrow \mathcal{V}, \Delta=(\mathfrak{t}, \mathfrak{s}) \in[0,6] \times[0,6]$ are specified in the next section.

\section{Preliminaries}

Let us consider $\mathcal{V}$ and $\mathcal{W}$ as real Banach spaces with respective norms $\|\cdot\|_{\mathcal{V}}$ and $\|\cdot\|_{\mathcal{W}}$. $\mathcal{C}(\mathfrak{J}, \mathcal{V})$ denotes the Banach space formed by all continuous functions from $\mathfrak{J}$ into $\mathcal{V}$ with corresponding norm function $\|w\|=\sup _{\mathfrak{t} \in \mathfrak{J}}\|w(\mathfrak{t})\| \cdot \mathcal{L}^{2}(\mathfrak{J}, \mathcal{W})$ denotes the Banach space of all $\mathcal{W}$-valued Bochner square integrable functions defined on $\mathfrak{J}$ w.r.t. norm function

$$
\|w\|=\left(\int_{0}^{6}\|w(\mathfrak{t})\|^{2} d \mathfrak{t}\right)^{\frac{1}{2}}, \quad w \in \mathcal{L}^{2}(\mathfrak{J}, \mathcal{W})
$$

Definition 2.1 ([20]) Hilfer fractional derivative of a continuously differentiable function $f$ of order $0<v<1$ and $0 \leq \mu \leq 1$ is defined as

$$
\mathcal{D}_{0^{+}}^{v, \mu} f(\mathfrak{t})=\mathcal{I}_{0^{+}}^{\nu(1-\mu)} \frac{d}{d \mathfrak{t}} \mathcal{I}_{1^{+}}^{(1-v)(1-\mu)} f(\mathfrak{t})=\mathcal{I}_{0^{+}}^{\nu(1-\mu)} \mathcal{D}_{0^{+}}^{\nu+\mu-v \mu} f(\mathfrak{t})
$$


where $\mathcal{I}_{0^{+}}^{\nu(1-\mu)}$ is a Riemann-Liouville fractional integral and $\mathcal{D}_{0^{+}}^{v+\mu-v \mu}$ is a RiemannLiouville fractional derivative.

Definition 2.2 ([38]) For $\alpha>0$, the Riemann-Liouville fractional integral of a continuously differentiable $f$ of order $\alpha$ is defined as follows:

$$
\mathcal{I}_{0^{+}}^{\alpha} f(\mathfrak{t})=\frac{1}{\Gamma(\alpha)} \int_{0}^{\mathfrak{t}}(\mathfrak{t}-\mathfrak{s})^{\alpha-1} f(\mathfrak{s}) d \mathfrak{s}
$$

Almost sectorial operator $([21,37])$ Let $0<\beta<\pi$ and $-1<\gamma<0$. We define $S_{\beta}^{o}=\{\omega \in$ $\mathbb{C} \backslash\{0\}:|\arg \omega|<\beta\}$ and $S_{\beta}=\bar{S}_{\beta}^{o}=\{\omega \in \mathbb{C} \backslash\{0\}:|\arg \omega| \leq \beta\} \cup\{0\}$. A closed linear operator $\mathcal{A}: D(\mathcal{A}) \subset \mathcal{V} \longrightarrow \mathcal{V}$ is called an almost sectorial operator if the following hold:

1. $\sigma(\mathcal{A})$ is contained in $S_{\omega}$.

2. $\forall \beta \in(\omega, \pi)$ there exists a constant $\mathcal{M}_{\beta}>0$ such that $\|\mathcal{R}(\mathfrak{z}, \mathcal{A})\|_{L(\mathcal{V})} \leq \mathcal{M}_{\beta}|\mathfrak{z}|^{\gamma}$, where $\mathcal{R}(\mathfrak{z}, \mathcal{A})=(\mathfrak{z} I-\mathcal{A})^{-1}$ is the resolvent operator of $\mathcal{A}$ for $\mathfrak{z} \in \rho(\mathcal{A})$.

Let us define $\Theta_{\omega}^{\gamma}$ as a family of almost sectorial operators.

Proposition 2.1 ([21]) Let $\mathcal{T}(\mathfrak{t})$ be the compact semigroup defined in [37] and $\mathcal{A} \in \Theta_{\omega}^{\gamma}$ for $-1<\gamma<0$ and $0<\omega<\frac{\pi}{2}$. Then following holds for $\mathcal{T}(\mathfrak{t})$ :

(i) $\mathcal{T}(\mathfrak{t})$ is analytic and $\frac{d^{n}}{d \mathfrak{t}^{n}} \mathcal{T}(\mathfrak{t})=(-\mathcal{A})^{n} \mathcal{T}(\mathfrak{t}), \mathfrak{t} \in S_{\frac{\pi}{2}-\omega}^{o}$. (ii) $\mathcal{T}(\mathfrak{t}+\mathfrak{s})=\mathcal{T}(\mathfrak{t}) \mathcal{T}(\mathfrak{s}) \forall \mathfrak{t}, \mathfrak{s} \in$ $S_{\frac{\pi}{2}-\omega}^{o}$. (iii) $\|\mathcal{T}(\mathfrak{t})\|_{L(\mathcal{V})} \leq c_{0} \mathfrak{t}^{-\gamma-1}, \mathfrak{t}>0$; where $c_{0}>0$ is a constant. (iv) Let $\Sigma_{\mathcal{T}}=\{v \in \mathcal{V}$ : $\left.\lim _{\mathfrak{t} \rightarrow 0^{+}} \mathcal{T}(\mathfrak{t}) v=v\right\}$, then $D\left(\mathcal{A}^{\theta}\right) \subset \Sigma_{\mathcal{T}}$ if $\theta>1+\gamma$.

Let us define the operator families $\left\{\mathcal{S}_{v}(\mathfrak{t})\right\}_{\mathfrak{t} \in S_{\frac{\pi}{2}-\omega}^{o}}$ and $\left\{Q_{V}(\mathfrak{t})\right\}_{\mathfrak{t} \in S_{\frac{\pi}{2}-\omega}^{o}}$ as follows:

$$
\begin{aligned}
& \mathcal{S}_{\nu}(\mathfrak{t})=\int_{0}^{\infty} \mathcal{M}_{v}(\xi) \mathcal{T}\left(\mathfrak{t}^{v} \xi\right) d \xi \\
& \mathcal{Q}_{\nu}(\mathfrak{t})=\int_{0}^{\infty} \nu \xi \mathcal{M}_{\nu}(\xi) \mathcal{T}\left(\mathfrak{t}^{v} \xi\right) d \xi
\end{aligned}
$$

where $\mathcal{M}_{v}(\theta)$ is a Wright-type function defined as follows:

$$
\mathcal{M}_{\nu}(\theta)=\sum_{n \in \mathbb{N}} \frac{(-\theta)^{n-1}}{\Gamma(1-v n)(n-1) !}, \quad \theta \in \mathbb{C} .
$$

Proposition $2.2([21])$ (i) For each fixed $\mathfrak{t} \in S_{\frac{\pi}{2}-\omega}^{o}, \mathcal{S}_{v}(\mathfrak{t})$ and $Q_{\nu}(\mathfrak{t})$ are bounded linear operators in $\mathcal{V}$. Also

$$
\left\|\mathcal{S}_{v}(\mathfrak{t})\right\| \leq C_{\mathcal{S}} \mathfrak{t}^{-v(1+\gamma)}, \quad\left\|Q_{v}(\mathfrak{t})\right\| \leq C_{\mathcal{P}} \mathfrak{t}^{-v(1+\gamma)}, \quad \mathfrak{t}>0,
$$

where $C_{S}$ and $C_{P}$ are constants depending only on $v$ and $\gamma$. (ii) $\mathcal{S}_{v}(\mathfrak{t})$ and $Q_{\nu}(\mathfrak{t})$ are continuous in the uniform operator topology for $\mathfrak{t}>0$. 
Lemma $2.1([2,20])$ The mild solution for the system of equations Eq. (1.1) is defined as follows:

$$
\begin{aligned}
v(\mathfrak{t})= & \mathcal{S}_{v, \mu}(\mathfrak{t}) \sum_{k=1}^{\mathfrak{m}} c_{k} v\left(\mathfrak{t}_{k}\right) \\
& +\int_{0}^{\mathfrak{t}} \mathcal{K}_{\psi}(\mathfrak{t}-\mathfrak{s})\left\{\Upsilon w(\mathfrak{s})+\Psi\left(\mathfrak{s}, v(\mathfrak{s}), \int_{0}^{\mathfrak{s}} \mathfrak{q}(\mathfrak{s}, \zeta) \varphi(\mathfrak{s}, \zeta, v(\zeta)) d \zeta\right)\right\} d \mathfrak{s}
\end{aligned}
$$

where $\mathcal{S}_{\nu, \mu}(\mathfrak{t})=\mathcal{I}_{0^{+}}^{\mu(1-v)} \mathcal{K}_{\nu}(\mathfrak{t})$ and $\mathcal{K}_{\nu}(\mathfrak{t})=\mathfrak{t}^{\nu-1} \mathcal{Q}_{\nu}(\mathfrak{t})$

Proposition $2.3([21])$ (i) For every fixed $\mathfrak{t} \in S_{\frac{\pi}{2}-\omega}^{o}, \mathcal{S}_{v, \mu}(\mathfrak{t})$ and $\mathcal{K}_{v}(\mathfrak{t})$ are bounded linear operators on $\mathcal{V}$. For $\mathfrak{t}>0$,

$$
\left\|\mathcal{K}_{v}(\mathfrak{t}) v\right\| \leq C_{P} \mathfrak{t}^{-1-\nu \gamma}\|v\|, \quad\left\|\mathcal{S}_{\nu, \mu}(\mathfrak{t}) v\right\| \leq \frac{\Gamma(-\gamma \nu)}{\Gamma(\mu(1-v)-v \gamma)} C_{P} \mathfrak{t}^{\mu(1-v)-v \gamma-1}\|v\| .
$$

(ii) $\mathcal{S}_{v, \mu}(\mathfrak{t})$ and $\mathcal{K}_{v}(\mathfrak{t})$ are strongly continuous operators.

Let $\mathcal{M}_{\mathcal{S}}=\sup _{\mathfrak{t} \in \mathfrak{J}^{\prime}}\left\|\mathcal{S}_{v, \mu}(\mathfrak{t})\right\|, \mathfrak{J}^{\prime}=(0,6]$. Assume that $\sum_{\kappa=1}^{\mathfrak{m}}\left|c_{K}\right| \leq \frac{1}{\mathfrak{M}_{\mathcal{S}}}$.

We have

$$
\left\|\sum_{k=1}^{\mathfrak{m}} c_{k} \mathcal{S}_{v, \mu}\left(\mathfrak{t}_{k}\right)\right\| \leq \mathcal{M}_{\mathcal{S}} \cdot \frac{1}{\mathcal{M}_{\mathcal{S}}}<1 .
$$

By the operator spectrum theorem, $\mathcal{O}=\left(I-\sum_{\kappa=1}^{\mathfrak{m}} c_{\kappa} \mathcal{S}_{v, \mu}\left(\mathfrak{t}_{k}\right)\right)^{-1}$ exists bounded and $D(\mathcal{O})=\mathcal{V}$.

By the Neumann series expression, $\mathcal{O}$ can be expressed as $\mathcal{O}=\sum_{n=0}^{\infty}\left[\sum_{k=1}^{\mathfrak{m}} c_{k} \mathcal{S}_{v, \mu}\left(\mathfrak{t}_{k}\right)\right]^{n}$.

Therefore

$$
\|\mathcal{O}\| \leq \frac{1}{1-\sum_{k=1}^{\mathfrak{m}}\left|c_{k}\right| \mathcal{M}_{\mathcal{S}}} .
$$

By Lemma 2.1 mild solutions of Eq. (1.1) are given by

$$
\begin{aligned}
& v(\mathfrak{t})=\mathcal{S}_{v, \mu}(\mathfrak{t}) \hat{v}+\int_{0}^{\mathfrak{t}} \mathcal{K}_{\nu}(\mathfrak{t}-\mathfrak{s})\left\{\Upsilon w(\mathfrak{s})+\Psi\left(\mathfrak{s}, v(\mathfrak{s}), \int_{0}^{\mathfrak{s}} \mathfrak{q}(\mathfrak{s}, \zeta) \varphi(\mathfrak{s}, \zeta, v(\zeta)) d \zeta\right)\right\} d \mathfrak{s}, \\
& \text { where } \hat{v}=\sum_{k=1}^{\mathfrak{m}} c_{k} v\left(\mathfrak{t}_{k}\right)
\end{aligned}
$$

At $\mathfrak{t}=\mathfrak{t}_{k}$,

$$
\begin{aligned}
& v\left(\mathfrak{t}_{k}\right)=\mathcal{S}_{v, \mu}(\mathfrak{t}) \hat{v}+\int_{0}^{\mathfrak{t}_{k}} \mathcal{K}_{\nu}\left(\mathfrak{t}_{\kappa}-\mathfrak{s}\right)\left\{\Upsilon w(\mathfrak{s})+\Psi\left(\mathfrak{s}, v(\mathfrak{s}), \int_{0}^{\mathfrak{s}} \mathfrak{q}(\mathfrak{s}, \zeta) \varphi(\mathfrak{s}, \zeta, v(\zeta)) d \zeta\right)\right\} d \mathfrak{s}, \\
& \sum_{k=1}^{\mathfrak{m}} c_{k} v\left(\mathfrak{t}_{k}\right)=\sum_{k=1}^{\mathfrak{m}} c_{k} \mathcal{S}_{v, \mu}(\mathfrak{t}) \hat{v}+\sum_{k=1}^{\mathfrak{m}} c_{k} \int_{0}^{\mathfrak{t}_{k}} \mathcal{K}_{v}\left(\mathfrak{t}_{k}-\mathfrak{s}\right) \\
& \times\left\{\Upsilon w(\mathfrak{s})+\Psi\left(\mathfrak{s}, v(\mathfrak{s}), \int_{0}^{\mathfrak{s}} \mathfrak{q}(\mathfrak{s}, \zeta) \varphi(\mathfrak{s}, \zeta, v(\zeta)) d \zeta\right)\right\} d \mathfrak{s}
\end{aligned}
$$




$$
\begin{aligned}
\Longrightarrow \hat{v}[I & \left.-\sum_{k=1}^{\mathfrak{m}} c_{k} \mathcal{S}_{v, \mu}\left(\mathfrak{t}_{k}\right)\right] \\
= & \sum_{k=1}^{\mathfrak{m}} c_{k} \int_{0}^{\mathfrak{t}_{k}} \mathcal{K}_{v}\left(\mathfrak{t}_{k}-\mathfrak{s}\right) \\
& \times\left\{\Upsilon w(\mathfrak{s})+\Psi\left(\mathfrak{s}, v(\mathfrak{s}), \int_{0}^{\mathfrak{s}} \mathfrak{q}(\mathfrak{s}, \zeta) \varphi(\mathfrak{s}, \zeta, v(\zeta)) d \zeta\right)\right\} d \mathfrak{s} \\
\Longrightarrow \quad \hat{v}= & \sum_{k=1}^{\mathfrak{m}} c_{k} \mathcal{O} \int_{0}^{\mathfrak{t}_{k}} \mathcal{K}_{v}\left(\mathfrak{t}_{k}-\mathfrak{s}\right) \\
& \times\left\{\Upsilon w(\mathfrak{s})+\Psi\left(\mathfrak{s}, v(\mathfrak{s}), \int_{0}^{\mathfrak{s}} \mathfrak{q}(\mathfrak{s}, \zeta) \varphi(\mathfrak{s}, \zeta, v(\zeta)) d \zeta\right)\right\} d \mathfrak{s} .
\end{aligned}
$$

Thus the mild solutions of Eq. (1.1) are defined as follows:

$$
\begin{aligned}
v(\mathfrak{t})= & \sum_{k=1}^{\mathfrak{m}} c_{k} \mathcal{S}_{v, \mu}(\mathfrak{t}) \mathcal{O} \int_{0}^{\mathfrak{t}_{k}} \mathcal{K}_{v}\left(\mathfrak{t}_{k}-\mathfrak{s}\right) \\
& \times\left\{\Upsilon w(\mathfrak{s})+\Psi\left(\mathfrak{s}, v(\mathfrak{s}), \int_{0}^{\mathfrak{s}} \mathfrak{q}(\mathfrak{s}, \zeta) \varphi(\mathfrak{s}, \zeta, v(\zeta)) d \zeta\right)\right\} d \mathfrak{s} \\
& +\int_{0}^{\mathfrak{t}} \mathcal{K}_{v}(t-s)\left\{\Upsilon w(\mathfrak{s})+\Psi\left(\mathfrak{s}, v(\mathfrak{s}), \int_{0}^{\mathfrak{s}} \mathfrak{q}(\mathfrak{s}, \zeta) \varphi(\mathfrak{s}, \zeta, v(\zeta)) d \zeta\right)\right\} d \mathfrak{s}
\end{aligned}
$$

Now we introduce the Green's function $\mathcal{G}(\mathfrak{t}, \mathfrak{s})$ as follows:

$$
\mathcal{G}(\mathfrak{t}, \mathfrak{s})=\sum_{\kappa=1}^{\mathfrak{m}} \chi_{\mathfrak{t}_{k}}(\mathfrak{s}) \mathcal{S}_{\nu, \mu}(\mathfrak{t}) \mathcal{O} K_{\nu}\left(\mathfrak{t}_{\kappa}-\mathfrak{s}\right)+\chi_{\mathfrak{t}}(\mathfrak{s}) \mathcal{K}_{\nu}(t-s),
$$

where

$$
\chi_{\mathfrak{t}_{k}}(\mathfrak{s})=\left\{\begin{array}{ll}
c_{k}, & \mathfrak{s} \in\left[0, \mathfrak{t}_{k}\right), \\
0, & \mathfrak{s} \in\left[\mathfrak{t}_{k}, 6\right),
\end{array} \quad \chi_{\mathfrak{t}}(\mathfrak{s})= \begin{cases}1, & \mathfrak{s} \in[0, \mathfrak{t}) \\
0, & \mathfrak{s} \in[\mathfrak{t}, 6)\end{cases}\right.
$$

The mild solutions of Eq. (1.1) in terms of the Green's function are expressed as

$$
v(\mathfrak{t})=\int_{0}^{6} \mathcal{G}(\mathfrak{t}, \mathfrak{s})\left\{\Upsilon w(\mathfrak{s})+\Psi\left(\mathfrak{s}, v(\mathfrak{s}), \int_{0}^{\mathfrak{s}} \mathfrak{q}(\mathfrak{s}, \zeta) \varphi(\mathfrak{s}, \zeta, v(\zeta)) d \zeta\right)\right\} d \mathfrak{s}, \quad \mathfrak{t} \in \mathfrak{J}
$$

In addition to the above-mentioned propositions and lemma, we assume here the following assumptions to establish the proposed results:

(A1) For each $\mathfrak{t} \in \mathfrak{J}^{\prime}, \Psi: \mathfrak{J}^{\prime} \times \mathcal{V} \times \mathcal{V} \rightarrow \mathcal{V}$ is a Caratheodory function.

(A2) There exist $\psi_{1} \in L^{1}\left(\mathfrak{J}, \mathbb{R}^{+}\right)$and a continuous function $\psi_{2}$ such that, for $v_{1}, v_{2} \in \mathcal{V}$ and $\mathfrak{t} \in \mathfrak{J}, \Psi\left(\mathfrak{t}, v_{1}, v_{2}\right) \leq \psi_{1}(\mathfrak{t})\left\|v_{1}\right\|+\psi_{2}(\mathfrak{t})\left\|\tau_{2}\right\|$.

(A3) $\varphi(\mathfrak{t}, \mathfrak{s}, \cdot): \mathcal{V} \longrightarrow \mathcal{V}$ is a Caratheodory function and there exists $w: \Delta \longrightarrow \mathbb{R}$ with

$$
\bar{w}=\sup _{t \in \mathfrak{J}} \int_{0}^{t} w(\mathfrak{t}, \mathfrak{s}) d \mathfrak{s}<\infty \quad \text { such that }\|\varphi(\mathfrak{t}, \mathfrak{s}, v)\| \leq w(\mathfrak{t}, \mathfrak{s})\|v\|, v \in \mathcal{V} .
$$


(A4) $\mathfrak{q}(t, \mathfrak{s})$ is bounded on $\mathfrak{J}=[0,6]$ with $\mathfrak{q}^{*}=\sup \{\mathfrak{q}(t, \mathfrak{s}): 0 \leq \mathfrak{s} \leq \mathfrak{t}\}<\infty$.

(A5) There exists $\kappa \in L^{1}\left(\mathfrak{J}, \mathbb{R}^{+}\right)$such that $\|\Upsilon w(\mathfrak{t})\| \leq \kappa(\mathfrak{t}), \forall \mathfrak{t} \in \mathfrak{J}$ and $w \in \mathcal{L}^{2}(\mathfrak{J}, \mathcal{W})$.

\section{Existence result}

For $\mathfrak{J}=[0,6]$ and $\mathfrak{J}^{\prime}=[0,6)$, we introduce

$$
\mathcal{Y}=\left\{v \in C\left(\mathfrak{J}^{\prime}, \mathcal{V}\right): \lim _{\mathfrak{t} \rightarrow 0} \mathfrak{t}^{(1+v \gamma)(1-\mu)} v(\mathfrak{t}) \text { exists and is finite }\right\}
$$

with the norm defined as $\|v\|_{\mathcal{Y}}=\sup _{\mathfrak{t} \in \mathcal{J}^{\prime}}\left\{\mathfrak{t}^{(1+\nu \gamma)(1-\mu)}\|v(\mathfrak{t})\|\right\}$.

Let $y(\mathfrak{t})=\mathfrak{t}^{(1+\nu \gamma)(1-\mu)} v(\mathfrak{t})$. Then $v \in \mathcal{Y}$ iff $y \in C(\mathfrak{J}, \mathcal{V})$ and $\|v\|_{\mathcal{Y}}=\|y\|$.

We define

$$
\mathcal{B}_{\delta}(\mathfrak{J})=\{y \in \mathcal{C}(\mathfrak{J}, \mathcal{V}):\|y\| \leq \delta\} \quad \text { and } \quad \mathcal{B}_{\delta}^{\mathcal{Y}}\left(\mathfrak{J}^{\prime}\right)=\left\{v \in \mathcal{Y}:\|v\|_{\mathcal{Y}} \leq \delta\right\}
$$

Define an operator $\mathcal{P}: \mathcal{B}_{\delta}(\mathfrak{J}) \longrightarrow \mathcal{B}_{\delta}(\mathfrak{J})$ such that

$$
\mathcal{P} y(\mathfrak{t})= \begin{cases}0, & \mathfrak{t}=0, \\ \mathfrak{t}^{(1+v \gamma)(1-\mu)} \Pi v(\mathfrak{t}), & \mathfrak{t} \in \mathfrak{J}^{\prime}=(0,6]\end{cases}
$$

where $\Pi: \mathcal{C}\left(\mathfrak{J}^{\prime}, \mathcal{V}\right) \longrightarrow C\left(\mathfrak{J}^{\prime}, \mathcal{V}\right)$ is defined as

$$
\Pi v(\mathfrak{t})=\int_{0}^{6} \mathcal{G}(\mathfrak{t}, \mathfrak{s})\left\{\Upsilon w(\mathfrak{s})+\Psi\left(\mathfrak{s}, v(\mathfrak{s}), \int_{0}^{\mathfrak{s}} \mathfrak{q}(\mathfrak{s}, \zeta) \varphi(\mathfrak{s}, \zeta, v(\zeta)) d \zeta\right)\right\} d \mathfrak{s}
$$

Let us denote $\mathcal{N}_{1}=\sup _{\mathfrak{t} \in \mathcal{J}^{\prime}}\left\{\psi_{1}(\mathfrak{t})\right\}, \quad \mathcal{N}_{2}=\sup _{\mathfrak{t} \in \mathcal{J}^{\mathfrak{J}}}\left\{\psi_{2}(\mathfrak{t})\right\}, \hat{\mathfrak{m}}=\sup _{\mathfrak{t} \in \mathcal{J}^{\prime}}\{\kappa(\mathfrak{t})\}$, and $\wedge_{o}=$ $\frac{\sum_{k=1}^{\mathfrak{m}}\left|c_{k}\right|}{1-\sum_{k=1}^{\mathrm{m}}\left|c_{K}\right| \mathcal{M}_{S}}$.

Lemma 3.1 Let $\mathcal{A} \in \Theta_{\omega}^{\gamma}$ for $-1<\gamma<0$ and $0<\omega<\frac{\pi}{2}$. Assume that assumptions (A1)-(A5) and Proposition 2.3 hold. Then the operator $\left\{\mathcal{P} y: y \in \mathcal{B}_{\delta}(\mathfrak{J})\right\}$ is bounded and continuous if $C_{P}\left(\mathcal{N}_{1}+\mathfrak{N}_{2} \bar{w} \mathfrak{q}^{*}\right)\left(1+\mathcal{M}_{\mathcal{S}} \wedge_{o}\right) 6^{-v \gamma}<1$.

Proof For $y \in \mathcal{B}_{\delta}(\mathfrak{J})$, we have

$$
\begin{aligned}
\|\mathcal{P} y\|= & \left\|\mathfrak{t}^{(1+\nu \gamma)(1-\mu)} \int_{0}^{6} \mathcal{G}(\mathfrak{t}, \mathfrak{s})\left\{\Upsilon w(\mathfrak{s})+\Psi\left(\mathfrak{s}, v(\mathfrak{s}), \int_{0}^{\mathfrak{s}} \mathfrak{q}(\mathfrak{s}, \zeta) \varphi(\mathfrak{s}, \zeta, v(\zeta)) d \zeta\right)\right\} d \mathfrak{s}\right\| \\
\leq & \| \mathfrak{t}^{(1+\nu \gamma)(1-\mu)} \sum_{\kappa=1}^{\mathfrak{m}} c_{k} \mathcal{S}_{v, \mu}(\mathfrak{t}) \mathcal{O} \int_{0}^{\mathfrak{t}_{k}} \mathcal{K}_{v}\left(\mathfrak{t}_{\kappa}-\mathfrak{s}\right) \\
& \times\left\{\Upsilon_{w(\mathfrak{s})}+\Psi\left(\mathfrak{s}, v(\mathfrak{s}), \int_{0}^{\mathfrak{s}} \mathfrak{q}(\mathfrak{s}, \zeta) \varphi(\mathfrak{s}, \zeta, v(\zeta)) d \zeta\right)\right\} d \mathfrak{s} \| \\
& +\| \mathfrak{t}^{(1+\nu \gamma)(1-\mu)} \int_{0}^{\mathfrak{t}} \mathcal{K}_{v}(t-s) \\
& \times\left\{\Upsilon_{w(\mathfrak{s})}+\Psi\left(\mathfrak{s}, v(\mathfrak{s}), \int_{0}^{\mathfrak{s}} \mathfrak{q}(\mathfrak{s}, \zeta) \varphi(\mathfrak{s}, \zeta, v(\zeta)) d \zeta\right)\right\} d \mathfrak{s} \| \\
\leq & \mathcal{M}_{\mathcal{S}} \wedge_{o} C_{\mathcal{P}} \mathfrak{t}^{(1+\nu \gamma)(1-\mu)} \int_{0}^{\mathfrak{t}_{\kappa}}\left(\mathfrak{t}_{\kappa}-\mathfrak{s}\right)^{-1-\nu \gamma}\left\{\psi_{1}(\mathfrak{s})\|v\|+\psi_{2}(\mathfrak{s}) \bar{w} \mathfrak{q}^{*}\|v\|+\kappa(\mathfrak{s})\right\} d \mathfrak{s}
\end{aligned}
$$




$$
\begin{aligned}
& +C_{P} \mathfrak{t}^{(1+\nu \gamma)(1-\mu)} \int_{0}^{\mathfrak{t}}(\mathfrak{t}-\mathfrak{s})^{-1-\nu \gamma}\left\{\psi_{1}(\mathfrak{s})\|v\|+\psi_{2}(\mathfrak{s}) \bar{w} \mathfrak{q}^{*}\|v\|+\kappa(\mathfrak{s})\right\} d \mathfrak{s} \\
\leq & C_{P} \delta\left(\mathcal{N}_{1}+\mathcal{N}_{2} \bar{w} \mathfrak{q}^{*}\right)\left(1+\mathcal{M}_{\mathcal{S}} \wedge_{o}\right) \frac{6^{-\nu \gamma}}{(-\nu \gamma)}+C_{P} \hat{\mathcal{M}}\left(1+\mathcal{M}_{\mathcal{S}} \wedge_{o}\right) \frac{6^{(1+\nu \gamma)(1-\mu)-\nu \gamma}}{(-\nu \gamma)} \\
\leq & C_{P} \delta\left(\mathcal{N}_{1}+\mathcal{N}_{2} \bar{w} q^{*}\right)\left(1+\mathcal{M}_{\mathcal{S}} \wedge_{o}\right) 6^{-\nu \gamma} \\
& +C_{P} \hat{\mathcal{M}}\left(1+\mathcal{M}_{\mathcal{S}} \wedge_{o}\right) 6^{(1+\nu \gamma)(1-\mu)-\nu \gamma} \quad \text { as }(-\nu \gamma)>0 .
\end{aligned}
$$

If possible, suppose $\|\mathcal{P} y\|>\delta$.

This implies

$$
C_{P}\left(\mathcal{N}_{1}+\mathcal{N}_{2} \bar{w} q^{*}\right)\left(1+\mathcal{M}_{\mathcal{S}} \wedge_{o}\right) b^{-\nu \gamma}+\frac{C_{P} \hat{\mathcal{M}}\left(1+\mathcal{M}_{\mathcal{S}} \wedge_{o}\right) 6^{(1+\nu \gamma)(1-\mu)-\nu \gamma}}{\delta}>1
$$

Applying $\lim _{\delta \rightarrow \infty}$ on both sides, we have

$$
C_{P}\left(\mathcal{N}_{1}+\mathcal{N}_{2} \bar{w} q^{*}\right)\left(1+\mathcal{M}_{\mathcal{S}} \wedge_{o}\right) 6^{-v \gamma}>1
$$

which is a contradiction.

So our supposition is wrong. Therefore $\|\mathcal{P} y\| \leq \delta$. This implies the boundedness of operator $\mathcal{P}$.

Now, to show that $\mathcal{P}: \mathcal{B}_{\delta}(\mathfrak{J}) \longrightarrow \mathcal{B}_{\delta}(\mathfrak{J})$ is a continuous operator, let $y_{n}$ and $y \in \mathcal{B}_{\delta}(\mathfrak{J})$ with $\lim _{n \rightarrow \infty} y_{n}=y, n \in \mathbb{N}$.

By the continuity of function $\Psi$ w.r.t. to the second and third variable

$$
\begin{aligned}
& \Psi\left(\mathfrak{t}, v_{n}(\mathfrak{t}), \int_{0}^{\mathfrak{t}} \mathfrak{q}(\mathfrak{t}, \mathfrak{s}) \varphi\left(\mathfrak{t}, \mathfrak{s}, v_{n}(\mathfrak{s})\right) d \mathfrak{s}\right) \\
& \quad \longrightarrow \Psi\left(\mathfrak{t}, v(\mathfrak{t}), \int_{0}^{\mathfrak{t}} \mathfrak{q}(\mathfrak{t}, \mathfrak{s}) \varphi(\mathfrak{t}, \mathfrak{s}, v(\mathfrak{s})) d \mathfrak{s}\right) \quad \text { as } n \longrightarrow \infty
\end{aligned}
$$

For $\mathfrak{t} \in \mathfrak{J}$,

$$
\begin{aligned}
\left\|\mathcal{P} y_{n}(\mathfrak{t})-\mathcal{P} y(\mathfrak{t})\right\| \\
\leq \mathcal{M}_{\mathfrak{s}} \wedge_{o} \mathfrak{t}^{(1+\nu \gamma)(1-\mu)} \int_{0}^{\mathfrak{t}_{k}}\left\|\mathcal{K}_{v}\left(\mathfrak{t}_{k}-\mathfrak{s}\right)\right\| \| \Psi\left(\mathfrak{s}, v_{n}(\mathfrak{s}), \int_{0}^{\mathfrak{s}} \mathfrak{q}(\mathfrak{s}, \zeta) \varphi\left(\mathfrak{s}, \zeta, v_{n}(\zeta)\right) d \zeta\right) \\
\quad-\Psi\left(\mathfrak{s}, v(\mathfrak{s}), \int_{0}^{\mathfrak{s}} \mathfrak{q}(\mathfrak{s}, \zeta) \varphi(\mathfrak{s}, \zeta, v(\zeta)) d \zeta\right) \| d \mathfrak{s} \\
\quad+\mathfrak{t}^{(1+\nu \gamma)(1-\mu)} \int_{0}^{\mathfrak{t}}\left\|\mathcal{K}_{v}(t-s)\right\| \| \Psi\left(\mathfrak{s}, v_{n}(\mathfrak{s}), \int_{0}^{\mathfrak{s}} \mathfrak{q}(\mathfrak{s}, \zeta) \varphi\left(\mathfrak{s}, \zeta, v_{n}(\zeta)\right) d \zeta\right) \\
\quad-\Psi\left(\mathfrak{s}, v(\mathfrak{s}), \int_{0}^{\mathfrak{s}} \mathfrak{q}(\mathfrak{s}, \zeta) \varphi(\mathfrak{s}, \zeta, v(\zeta)) d \zeta\right) \| d \mathfrak{s} \\
\leq \mathcal{M}_{\mathfrak{s}} \wedge_{o} \mathfrak{t}^{(1+\nu \gamma)(1-\mu)} C_{P} \int_{0}^{\mathfrak{t}_{k}}\left(\mathfrak{t}_{k}-\mathfrak{s}\right)^{-1-\nu \gamma} \| \Psi\left(\mathfrak{s}, v_{n}(\mathfrak{s}), \int_{0}^{\mathfrak{s}} \mathfrak{q}(\mathfrak{s}, \zeta) \varphi\left(\mathfrak{s}, \zeta, v_{n}(\zeta)\right) d \zeta\right) \\
\quad-\Psi\left(\mathfrak{s}, v(\mathfrak{s}), \int_{0}^{\mathfrak{s}} \mathfrak{q}(\mathfrak{s}, \zeta) \varphi(\mathfrak{s}, \zeta, v(\zeta)) d \zeta\right) \| d \mathfrak{s}
\end{aligned}
$$




$$
\begin{aligned}
& +\mathfrak{t}^{(1+\nu \gamma)(1-\mu)} C_{P} \int_{0}^{\mathfrak{t}}(t-s)^{-1-v \gamma} \| \Psi\left(\mathfrak{s}, v_{n}(\mathfrak{s}), \int_{0}^{\mathfrak{s}} \mathfrak{q}(\mathfrak{s}, \zeta) \varphi\left(\mathfrak{s}, \zeta, v_{n}(\zeta)\right) d \zeta\right) \\
& -\Psi\left(\mathfrak{s}, v(\mathfrak{s}), \int_{0}^{\mathfrak{s}} \mathfrak{q}(\mathfrak{s}, \zeta) \varphi(\mathfrak{s}, \zeta, v(\zeta)) d \zeta\right) \| d \mathfrak{s} .
\end{aligned}
$$

Since

$$
\begin{aligned}
& \mathfrak{t}^{(1+\nu \gamma)(1-\mu)} \| \Psi\left(\mathfrak{s}, v_{n}(\mathfrak{s}), \int_{0}^{\mathfrak{s}} \mathfrak{q}(\mathfrak{s}, \zeta) \varphi\left(\mathfrak{s}, \zeta, v_{n}(\zeta)\right) d \zeta\right) \\
& -\Psi\left(\mathfrak{s}, v(\mathfrak{s}), \int_{0}^{\mathfrak{s}} \mathfrak{q}(\mathfrak{s}, \zeta) \varphi(\mathfrak{s}, \zeta, v(\zeta)) d \zeta\right) \| \leq 2 \delta\left(\mathcal{N}_{1}+\mathcal{N}_{2} \bar{w} \mathfrak{q}^{*}\right), \quad \text { which is finite, }
\end{aligned}
$$

by the Lebesgue dominated convergence theorem and the continuity of function $\Psi$,

$$
\left\|\mathcal{P} y_{n}(\mathfrak{t})-\mathcal{P} y(\mathfrak{t})\right\| \longrightarrow 0 \quad \text { as } n \longrightarrow \infty
$$

This completes the proof of Lemma 3.1.

Lemma 3.2 Let $\mathcal{A} \in \Theta_{\omega}^{\gamma}$ for $-1<\gamma<0$ and $0<\omega<\frac{\pi}{2}$. If assumptions (A1)-(A5) and Proposition 2.3 are satisfied, the operator $\left\{\mathcal{P} y: y \in \mathcal{B}_{\delta}(\mathfrak{J})\right\}$ is equicontinuous for $\mathfrak{t} \in \mathfrak{J}$.

Proof For $y \in \mathcal{B}_{\delta}(\mathfrak{J})$ and $0 \leq \mathfrak{t}_{1}<\mathfrak{t}_{2}<b$,

$$
\begin{aligned}
& \left\|\mathcal{P} y\left(\mathfrak{t}_{2}\right)-\mathcal{P} y\left(\mathfrak{t}_{1}\right)\right\| \\
& =\left\|\mathfrak{t}_{2}^{(1+\nu \gamma)(1-\mu)} \Pi v\left(\mathfrak{t}_{2}\right)-\mathfrak{t}_{1}^{(1+\nu \gamma)(1-\mu)} \Pi v\left(\mathfrak{t}_{1}\right)\right\| \\
& =\| \mathfrak{t}_{2}^{(1+\nu \gamma)(1-\mu)} \int_{0}^{6} \mathcal{G}\left(\mathfrak{t}_{2}, \mathfrak{s}\right)\left\{\Upsilon w(\mathfrak{s})+\Psi\left(\mathfrak{s}, v(\mathfrak{s}), \int_{0}^{\mathfrak{s}} \mathfrak{q}(\mathfrak{s}, \zeta) \varphi(\mathfrak{s}, \zeta, v(\zeta)) d \zeta\right)\right\} d \mathfrak{s} \\
& -\mathfrak{t}_{1}^{(1+\nu \gamma)(1-\mu)} \int_{0}^{6} \mathcal{G}\left(\mathfrak{t}_{1}, \mathfrak{s}\right)\left\{\Upsilon w(\mathfrak{s})+\Psi\left(\mathfrak{s}, v(\mathfrak{s}), \int_{0}^{\mathfrak{s}} \mathfrak{q}(\mathfrak{s}, \zeta) \varphi(\mathfrak{s}, \zeta, v(\zeta)) d \zeta\right)\right\} d \mathfrak{s} \| . \\
& \leq \| \mathfrak{t}_{2}^{(1+\nu \gamma)(1-\mu)} \sum_{k=1}^{\mathfrak{m}} \mathcal{c}_{k} \mathcal{S}_{v, \mu}\left(\mathfrak{t}_{2}\right) \mathcal{O} \int_{0}^{\mathfrak{t}_{\kappa}} \mathcal{K}_{v}\left(\mathfrak{t}_{k}-\mathfrak{s}\right) \\
& \times\left\{\Upsilon w(\mathfrak{s})+\Psi\left(\mathfrak{s}, v(\mathfrak{s}), \int_{0}^{\mathfrak{s}} \mathfrak{q}(\mathfrak{s}, \zeta) \varphi(\mathfrak{s}, \zeta, v(\zeta)) d \zeta\right)\right\} d \mathfrak{s} \\
& -\mathfrak{t}_{1}^{(1+\nu \gamma)(1-\mu)} \sum_{k=1}^{\mathfrak{m}} \mathcal{c}_{k} \mathcal{S}_{\nu, \mu}\left(\mathfrak{t}_{1}\right) \mathcal{O} \int_{0}^{\mathfrak{t}_{k}} \mathcal{K}_{\nu}\left(\mathfrak{t}_{k}-\mathfrak{s}\right) \\
& \times\left\{\Upsilon w(\mathfrak{s})+\Psi\left(\mathfrak{s}, v(\mathfrak{s}), \int_{0}^{\mathfrak{s}} \mathfrak{q}(\mathfrak{s}, \zeta) \varphi(\mathfrak{s}, \zeta, v(\zeta)) d \zeta\right)\right\} d \mathfrak{s} \| \\
& +\| \mathfrak{t}_{2}^{(1+\nu \gamma)(1-\mu)} \int_{0}^{\mathfrak{t}_{1}} \mathcal{K}_{\nu}\left(\mathfrak{t}_{2}-\mathfrak{s}\right) \\
& \times\left\{\Upsilon w(\mathfrak{s})+\Psi\left(\mathfrak{s}, v(\mathfrak{s}), \int_{0}^{\mathfrak{s}} \mathfrak{q}(\mathfrak{s}, \zeta) \varphi(\mathfrak{s}, \zeta, v(\zeta)) d \zeta\right)\right\} d \mathfrak{s} \\
& -\mathfrak{t}_{1}^{(1+\nu \gamma)(1-\mu)} \int_{0}^{\mathfrak{t}_{1}} \mathcal{K}_{v}\left(\mathfrak{t}_{\mathbf{1}}-\mathfrak{s}\right)
\end{aligned}
$$




$$
\begin{aligned}
& \quad \times\left\{\Upsilon w(\mathfrak{s})+\Psi\left(\mathfrak{s}, v(\mathfrak{s}), \int_{0}^{\mathfrak{s}} \mathfrak{q}(\mathfrak{s}, \zeta) \varphi(\mathfrak{s}, \zeta, v(\zeta)) d \zeta\right)\right\} d \mathfrak{s} \| \\
& +\| \mathfrak{t}_{2}^{(1+v \gamma)(1-\mu)} \int_{\mathfrak{t}_{1}}^{\mathfrak{t}_{2}} \mathcal{K}_{\nu}\left(\mathfrak{t}_{2}-\mathfrak{s}\right) \\
& \quad \times\left\{\Upsilon w(\mathfrak{s})+\Psi\left(\mathfrak{s}, v(\mathfrak{s}), \int_{0}^{\mathfrak{s}} \mathfrak{q}(\mathfrak{s}, \zeta) \varphi(\mathfrak{s}, \zeta, v(\zeta)) d \zeta\right)\right\} d \mathfrak{s} \| \\
& =I_{1}+I_{2}+I_{3} .
\end{aligned}
$$

Here,

$$
\begin{aligned}
I_{1}= & \| \mathfrak{t}_{2}^{(1+\nu \gamma)(1-\mu)} \sum_{k=1}^{\mathfrak{m}} c_{k} \mathcal{S}_{v, \mu}\left(\mathfrak{t}_{2}\right) \mathcal{O} \int_{0}^{\mathfrak{t}_{k}} \mathcal{K}_{\mathfrak{v}}\left(\mathfrak{t}_{k}-\mathfrak{s}\right) \\
& \times\left\{\Upsilon w(\mathfrak{s})+\Psi\left(\mathfrak{s}, v(\mathfrak{s}), \int_{0}^{\mathfrak{s}} \mathfrak{q}(\mathfrak{s}, \zeta) \varphi(\mathfrak{s}, \zeta, v(\zeta)) d \zeta\right)\right\} d \mathfrak{s} \\
& -\mathfrak{t}_{1}^{(1+\nu \gamma)(1-\mu)} \sum_{k=1}^{\mathfrak{m}} c_{k} \mathcal{S}_{v, \mu}\left(\mathfrak{t}_{1}\right) \mathcal{O} \int_{0}^{\mathfrak{t}_{k}} \mathcal{K}_{v}\left(\mathfrak{t}_{k}-\mathfrak{s}\right) \\
& \times\left\{\Upsilon_{w(\mathfrak{s})}+\Psi\left(\mathfrak{s}, v(\mathfrak{s}), \int_{0}^{\mathfrak{s}} \mathfrak{q}(\mathfrak{s}, \zeta) \varphi(\mathfrak{s}, \zeta, v(\zeta)) d \zeta\right)\right\} d \mathfrak{s} \| .
\end{aligned}
$$

The strong continuity of operator $\mathcal{S}_{v, \mu}(\mathfrak{t})$ yields $I_{1} \longrightarrow 0$ as $\mathfrak{t}_{1} \longrightarrow \mathfrak{t}_{2}$.

$$
\begin{aligned}
I_{2}= & \| \mathfrak{t}_{2}^{(1+\nu \gamma)(1-\mu)} \int_{0}^{\mathfrak{t}_{1}} \mathcal{K}_{v}\left(\mathfrak{t}_{2}-\mathfrak{s}\right) \\
& \times\left\{\Upsilon w(\mathfrak{s})+\Psi\left(\mathfrak{s}, v(\mathfrak{s}), \int_{0}^{\mathfrak{s}} \mathfrak{q}(\mathfrak{s}, \zeta) \varphi(\mathfrak{s}, \zeta, v(\zeta)) d \zeta\right)\right\} d \mathfrak{s} \\
& -\mathfrak{t}_{1}^{(1+\nu \gamma)(1-\mu)} \int_{0}^{\mathfrak{t}_{1}} \mathcal{K}_{v}\left(\mathfrak{t}_{1}-\mathfrak{s}\right) \\
& \times\left\{\Upsilon w(\mathfrak{s})+\Psi\left(\mathfrak{s}, v(\mathfrak{s}), \int_{0}^{\mathfrak{s}} \mathfrak{q}(\mathfrak{s}, \zeta) \varphi(\mathfrak{s}, \zeta, v(\zeta)) d \zeta\right)\right\} d \mathfrak{s} \| .
\end{aligned}
$$

Following Lemma 3.1 and assumptions (A1)-(A5), it is easy to see that

$$
\left\|\int_{0}^{\mathfrak{t}_{1}} \mathcal{K}_{\nu}\left(\mathfrak{t}_{\mathfrak{i}}-\mathfrak{s}\right)\left\{\Upsilon w(\mathfrak{s})+\Psi\left(\mathfrak{s}, v(\mathfrak{s}), \int_{0}^{\mathfrak{s}} \mathfrak{q}(\mathfrak{s}, \zeta) \varphi(\mathfrak{s}, \zeta, v(\zeta)) d \zeta\right)\right\} d \mathfrak{s}\right\|, \quad i=1,2
$$

exists and is bounded. Thus $I_{2} \longrightarrow 0$ as $\mathfrak{t}_{2} \longrightarrow \mathfrak{t}_{1}$.

$$
I_{3}=\left\|\mathfrak{t}_{2}^{(1+\nu \gamma)(1-\mu)} \int_{\mathfrak{t}_{1}}^{\mathfrak{t}_{2}} \mathcal{K}_{v}\left(\mathfrak{t}_{2}-\mathfrak{s}\right)\left\{\Upsilon w(\mathfrak{s})+\Psi\left(\mathfrak{s}, v(\mathfrak{s}), \int_{0}^{\mathfrak{s}} \mathfrak{q}(\mathfrak{s}, \zeta) \varphi(\mathfrak{s}, \zeta, v(\zeta)) d \zeta\right)\right\} d \mathfrak{s}\right\| .
$$

After following the given assumptions and performing some steps of calculation, we have

$$
I_{3} \leq \mathfrak{t}_{2}^{(1+\nu \gamma)(1-\mu)} C_{P} \delta\left(\mathcal{N}_{1}+\mathfrak{N}_{2} \bar{w} \mathfrak{q}^{*}\right)\left(\mathfrak{t}_{2}-\mathfrak{t}_{1}\right)^{-\gamma \nu}+\mathfrak{t}_{2}^{(1+\nu \gamma)(1-\mu)} C_{P} \hat{\mathcal{M}}\left(\mathfrak{t}_{2}-\mathfrak{t}_{1}\right)^{-\gamma \nu}
$$

Clearly, $I_{3} \longrightarrow 0$ as $\mathfrak{t}_{2} \longrightarrow \mathfrak{t}_{1}$. 
So, we have

$$
\left\|\mathcal{P} y\left(\mathfrak{t}_{2}\right)-\mathcal{P} y\left(\mathfrak{t}_{1}\right)\right\| \longrightarrow 0 \quad \text { as } \mathfrak{t}_{2} \longrightarrow t_{1}
$$

This proves the equicontinuity of operator $\mathcal{P} y(\mathfrak{t})$.

Theorem 3.1 Let $-1<\gamma<0,0<\omega<\frac{\pi}{2}$, and $\mathcal{A} \in \Theta_{\omega}^{\gamma}$. The system of equations Eq. (1.1) has at least one mild solution in $\mathcal{B}_{\delta}^{\gamma}\left(\mathfrak{J}^{\prime}\right)$ if assumptions (A1)-(A5) hold along with Lemma 3.1 and Lemma 3.2.

Proof The mild solution of Eq. (1.1) is equivalent to the fixed point of operator $\mathcal{P}$ : $\mathcal{B}_{\delta}(\mathfrak{J}) \longrightarrow \mathcal{B}_{\delta}(\mathfrak{J})$. Here we prove that the operator $\mathcal{P}$ has at least one fixed point.

In the following, we show that the operator $\mathcal{P}$ is relatively compact in $\mathcal{V}$ for every $\mathfrak{t} \in \mathfrak{J}$. We prove this by showing that there is a relatively compact set $\left\{\mathcal{P}^{\epsilon} y(\mathfrak{t}): y \in \mathcal{B}_{\delta}(\mathfrak{J})\right\}$ arbitrarily close to the set $\left\{\mathcal{P} y(\mathfrak{t}): y \in \mathcal{B}_{\delta}(\mathfrak{J})\right\}$ in $\mathcal{V}$ for $0<\mathfrak{t} \leq 6$

Define the operator $\mathcal{P}^{\epsilon} y$ by

$$
\begin{aligned}
\mathcal{P}^{\epsilon} y(\mathfrak{t})= & \mathfrak{t}^{(1+\nu \gamma)(1-\mu)} \sum_{\kappa=1}^{\mathfrak{m}} c_{k} \mathcal{S}_{\nu, \mu}(\mathfrak{t}) \mathcal{O} \int_{0}^{\mathfrak{t}_{k}} \mathcal{K}_{v}\left(\mathfrak{t}_{\kappa}-\mathfrak{s}\right) \\
& \times\left\{\Upsilon w(\mathfrak{s})+\Psi\left(\mathfrak{s}, v(\mathfrak{s}), \int_{0}^{\mathfrak{s}} \mathfrak{q}(\mathfrak{s}, \zeta) \varphi(\mathfrak{s}, \zeta, v(\zeta)) d \zeta\right)\right\} d \mathfrak{s} \\
& +\mathfrak{t}^{(1+\nu \gamma)(1-\mu)} \mathcal{K}_{v}(\epsilon) \int_{0}^{\mathfrak{t}-\epsilon} \mathcal{K}_{\nu}(t-\epsilon-s) \\
& \times\left\{\Upsilon w(\mathfrak{s})+\Psi\left(\mathfrak{s}, v(\mathfrak{s}), \int_{0}^{\mathfrak{s}} \mathfrak{q}(\mathfrak{s}, \zeta) \varphi(\mathfrak{s}, \zeta, v(\zeta)) d \zeta\right)\right\} d \mathfrak{s} .
\end{aligned}
$$

Since $\mathcal{S}_{\nu, \mu}(\mathfrak{t})$ and $\mathcal{K}_{v}(\mathfrak{t})$ are compact for $\mathfrak{t}>0$, the set $\mathcal{P}^{\epsilon} y(\mathfrak{t})$ is compact for $\mathfrak{t} \in \mathfrak{J}^{\prime}$. In the following

$$
\begin{aligned}
& \left\|\mathcal{P}^{\epsilon} y(\mathfrak{t})-\mathcal{P} y(\mathfrak{t})\right\| \\
& \leq \| \mathfrak{t}^{(1+\nu \gamma)(1-\mu)} \int_{0}^{\mathfrak{t}-\epsilon}\left\{\mathcal{K}_{\nu}(t-\epsilon-s) \mathcal{K}_{\nu}(\epsilon)-\mathcal{K}_{\nu}(\mathfrak{t}-\mathfrak{s})\right\} \\
& \times\left\{\Upsilon w(\mathfrak{s})+\Psi\left(\mathfrak{s}, v(\mathfrak{s}), \int_{0}^{\mathfrak{s}} \mathfrak{q}(\mathfrak{s}, \zeta) \varphi(\mathfrak{s}, \zeta, v(\zeta)) d \zeta\right)\right\} d \mathfrak{s} \| \\
& +\| \mathfrak{t}^{(1+\nu \gamma)(1-\mu)} \int_{\mathfrak{t}-\epsilon}^{\mathfrak{t}} \mathcal{K}_{\nu}(t-s) \\
& \times\left\{\Upsilon w(\mathfrak{s})+\Psi\left(\mathfrak{s}, v(\mathfrak{s}), \int_{0}^{\mathfrak{s}} \mathfrak{q}(\mathfrak{s}, \zeta) \varphi(\mathfrak{s}, \zeta, v(\zeta)) d \zeta\right)\right\} d \mathfrak{s} \| \\
& \leq \int_{0}^{\mathfrak{t}-\epsilon}\left\|\mathcal{K}_{\nu}(t-\epsilon-s) \mathcal{K}_{\nu}(\epsilon)-\mathcal{K}_{\nu}(\mathfrak{t}-\mathfrak{s})\right\|\left(\mathcal{N}_{1}+\mathcal{N}_{2} \bar{w} \mathfrak{q}^{*}\right) \delta d \mathfrak{s} \\
& +\sigma^{(1+\nu \gamma)(1-\mu)} \hat{\mathcal{M}} \int_{0}^{\mathfrak{t}-\epsilon}\left\|\mathcal{K}_{\nu}(t-\epsilon-s) \mathcal{K}_{\nu}(\epsilon)-\mathcal{K}_{\nu}(\mathfrak{t}-\mathfrak{s})\right\| d \mathfrak{s} \\
& +C_{p} \delta\left(\mathcal{N}_{1}+\mathcal{N}_{2} \bar{w} q^{*}\right) \frac{\epsilon^{-v \gamma}}{(-v \gamma)}+\hat{\mathcal{M}} C_{P} \sigma^{(1+\nu \gamma)(1-\mu)} \frac{\epsilon^{-v \gamma}}{(-v \gamma)}, \quad(-v \gamma)>0
\end{aligned}
$$


Clearly,

$$
\left\|\mathcal{P}^{\epsilon} y(\mathfrak{t})-\mathcal{P} y(\mathfrak{t})\right\| \longrightarrow 0 \quad \text { as } \epsilon \longrightarrow 0
$$

We have shown that the set $\left\{\mathcal{P} y(\mathfrak{t}): y \in \mathcal{B}_{\delta}(\mathfrak{J})\right\}$ is arbitrarily close to the relatively compact set $\left\{\mathcal{P}^{\epsilon} y(\mathfrak{t}): y \in \mathcal{B}_{\delta}(\mathfrak{J})\right\}$. This implies $\left\{\mathcal{P} y(\mathfrak{t}): y \in \mathcal{B}_{\delta}\left(\mathfrak{J}^{\prime}\right)\right\}$ is relatively compact in $\mathcal{V}$. Also, by Lemma 3.1 and Lemma 3.2, the operator $\mathcal{P}: \mathcal{B}_{\delta}\left(\mathfrak{J}^{\prime}\right) \longrightarrow \mathcal{B}_{\delta}\left(\mathfrak{J}^{\prime}\right)$ is bounded, continuous, and equicontinuous in $\mathcal{V}$. So by the Arzela-Ascoli theorem, $\left\{\mathcal{P} y(\mathfrak{t}): y \in \mathcal{B}_{\delta}\left(\mathfrak{J}^{\prime}\right)\right\}$ is a compact operator. Hence, by Schauder's fixed point theorem, $\mathcal{P}$ has at least one fixed point $y^{*} \in$ $B_{\delta}\left(\mathfrak{J}^{\prime}\right)$.

Let $v^{*}(\mathfrak{t})=\mathfrak{t}^{(1+\nu \gamma)(\mu-1)} y^{*}(\mathfrak{t})$. Then $v^{*}$ is a mild solution of Eq. (1.1). This completes the proof.

\section{Approximate controllability}

In this section, we discuss the approximate controllability of Eq. (1.1).

The system of equations Eq. (1.1) is said to be approximate controllable on $[0,6]$ if, for every desired final state $v_{6} \in \mathcal{V}$ and $\epsilon>0$, there exists a control function $w \in L^{2}(\mathfrak{J}, \mathcal{W})$ such that the mild solution ' $v^{\prime}$ of Eq. (1.1) satisfies

$$
\left\|v(6)-v_{6}\right\|<\epsilon .
$$

Following this, we first introduce the following two operators:

$$
\begin{aligned}
& \Gamma_{0}^{6}=\int_{0}^{6} \mathcal{G}(6, \mathfrak{s}) \Upsilon \Upsilon^{*} \mathcal{G}^{*}(6, \mathfrak{s}) d \mathfrak{s}, \\
& \mathcal{R}\left(\lambda, \Gamma_{0}^{6}\right)=\left(\lambda I+\Gamma_{0}^{6}\right)^{-1}, \quad \lambda>0,
\end{aligned}
$$

where $\Upsilon^{*}, \mathcal{O}^{*}, \mathfrak{K}_{\psi}^{*}(\mathfrak{t})$, and $\mathcal{S}_{v, \mu}^{*}(\mathfrak{t})$ characterize the adjoint operators of $\Upsilon, \mathcal{O}, \mathcal{K}_{v}(\mathfrak{t})$, and $\mathcal{S}_{v, \mu}(\mathfrak{t})$ respectively,

$$
\mathcal{G}^{*}(6, \mathfrak{s})=\sum_{k=1}^{\mathfrak{m}} \chi_{\mathfrak{t}_{k}}(\mathfrak{s}) \mathcal{S}_{\nu, \mu}^{*}(6) \mathcal{O}^{*} \mathcal{K}_{\psi}^{*}\left(\mathfrak{t}_{\kappa}-\mathfrak{s}\right)+\chi_{\mathfrak{t}}(\mathfrak{s}) \mathcal{K}_{\psi}^{*}(6-\mathfrak{s}), \quad \mathfrak{s} \in[0,6] .
$$

Theorem 4.1 Let $\mathcal{A} \in \Theta_{\omega}^{\gamma}$ for $-1<\gamma<0$ and $0<\omega<\frac{\pi}{2}$. Assume that assumptions (A1)(A5) and Proposition 2.3 hold. The system of equations Eq. (1.1) is approximate controllable on $\mathfrak{J}$ if $\lambda \mathcal{R}\left(\lambda, \Gamma_{0}^{6}\right) \longrightarrow 0$ as $\lambda \longrightarrow 0^{+}$in the strong operator topology.

Proof By Theorem 3.1, the system of equations Eq. (1.1) has at least one mild solution $v_{\lambda} \in \mathcal{B}_{\delta}$ given by

$$
v_{\lambda}(\mathfrak{t})=\int_{0}^{6} \mathcal{G}(\mathfrak{t}, \mathfrak{s})\left\{\Upsilon w_{\lambda}(\mathfrak{s})+\Psi\left(\mathfrak{s}, v_{\lambda}(\mathfrak{s}), \int_{0}^{\mathfrak{s}} \mathfrak{q}(\mathfrak{s}, \zeta) \varphi\left(\mathfrak{s}, \zeta, v_{\lambda}(\zeta)\right) d \zeta\right)\right\} d \mathfrak{s}, \quad \mathfrak{t} \in \mathfrak{J}
$$

where we define the control function $w_{\lambda}$ as

$$
w_{\lambda}(\mathfrak{t})=\Upsilon^{*} \mathcal{G}^{*}(6, \mathfrak{t}) \mathcal{R}\left(\lambda, \Gamma_{0}^{6}\right) \vartheta(v(\cdot))
$$


with

$$
\vartheta(v(\cdot))=v_{6}-\int_{0}^{6} \mathcal{G}(6, \mathfrak{s}) \Psi\left(\mathfrak{s}, v(\mathfrak{s}), \int_{0}^{\mathfrak{s}} \mathfrak{q}(\mathfrak{s}, \zeta) \varphi(\mathfrak{s}, \zeta, v(\zeta)) d \zeta\right) d \mathfrak{s} .
$$

At $\mathfrak{t}=6$,

$$
\begin{aligned}
v_{\lambda}(6) & =\int_{0}^{6} \mathcal{G}(6, \mathfrak{s})\left\{\Upsilon w_{\lambda}(\mathfrak{s})+\Psi\left(\mathfrak{s}, v_{\lambda}(\mathfrak{s}), \int_{0}^{\mathfrak{s}} \mathfrak{q}(\mathfrak{s}, \zeta) \varphi\left(\mathfrak{s}, \zeta, v_{\lambda}(\zeta)\right) d \zeta\right)\right\} d \mathfrak{s} \\
& =v_{6}-\vartheta\left(v_{\lambda}(\cdot)\right)+\int_{0}^{6} \mathcal{G}(6, \mathfrak{s}) \Upsilon w_{\lambda}(\mathfrak{s}) d \mathfrak{s} \\
& =v_{6}-\vartheta\left(v_{\lambda}(\cdot)\right)+\int_{0}^{6} \mathcal{G}(6, \mathfrak{s}) \Upsilon \Upsilon^{*} \mathcal{G}^{*}(6, \mathfrak{s}) \mathcal{R}\left(\lambda, \Gamma_{0}^{6}\right) \vartheta\left(v_{\lambda}(\cdot)\right) d \mathfrak{s} \\
& =v_{6}-\left(\lambda I+\Gamma_{0}^{6}\right) \cdot \frac{1}{\left(\lambda I+\Gamma_{0}^{6}\right)} \vartheta\left(v_{\lambda}(\cdot)\right)+\Gamma_{0}^{6} \mathcal{R}\left(\lambda, \Gamma_{0}^{6}\right) \vartheta\left(v_{\lambda}(\cdot)\right) \\
& =v_{6}-\left(\lambda I+\Gamma_{0}^{6}\right) \mathcal{R}\left(\lambda, \Gamma_{0}^{6}\right) \vartheta\left(v_{\lambda}(\cdot)\right)+\Gamma_{0}^{6} \mathcal{R}\left(\lambda, \Gamma_{0}^{6}\right) \vartheta\left(v_{\lambda}(\cdot)\right) \\
\Longrightarrow & v_{\lambda}(6)=v_{6}-\lambda \mathcal{R}\left(\lambda, \Gamma_{0}^{6}\right) \vartheta\left(v_{\lambda}(\cdot)\right), \\
\left(\int_{0}^{6} \|\right. & \left.\Psi\left(\mathfrak{s}, v_{\lambda}(\mathfrak{s}), \int_{0}^{\mathfrak{s}} \mathfrak{q}(\mathfrak{s}, \zeta) \varphi(\mathfrak{s}, \zeta, v(\zeta)) d \zeta\right) \|^{2}\right)^{\frac{1}{2}} \\
\leq & \left(\int_{0}^{6}\left(\psi_{1}(\mathfrak{s})\left\|v_{\lambda}\right\|+\psi_{2}(\mathfrak{s}) \bar{w} \mathfrak{q}^{*}\left\|v_{\lambda}\right\|\right)^{2} d \mathfrak{s}\right)^{\frac{1}{2}} \\
\leq & \left(\int_{0}^{6}\left(\psi_{1}(\mathfrak{s}) \delta+\psi_{2}(\mathfrak{s}) \bar{w} \mathfrak{q}^{*} \delta\right)^{2} d \mathfrak{s}\right)^{\frac{1}{2}} \\
\leq & \sigma^{\frac{1}{2}} \delta\left(\mathcal{N}_{1}^{2}+\mathcal{N}_{2}^{2} \bar{w}^{2} \mathfrak{q}^{* 2}+2 \mathfrak{\mathcal { N }} \mathfrak{\mathcal { N }}\left(\bar{w} \bar{w} \mathfrak{q}^{*}\right)^{\frac{1}{2}},\right.
\end{aligned}
$$

which implies that the sequence $\left\{\Psi\left(\cdot, v_{\lambda}(\cdot), \cdot\right): \lambda>0\right\}$ is bounded in the Hilbert space $L^{2}(\mathfrak{J}, \mathcal{W})$. Therefore, there exists a subsequence of the sequence $\left\{\Psi\left(\cdot, v_{\lambda}(\cdot), \cdot\right): \lambda>0\right\}$ converging weakly to some point $\varrho(\cdot) \in L^{2}(\mathfrak{J}, \mathcal{W})$.

Let us write

$$
\varpi=v_{6}-\int_{0}^{6} \mathcal{G}(6, \mathfrak{s}) \varrho(\mathfrak{s}) d \mathfrak{s} .
$$

Hence, by Eqs. (4.4) and (4.6), we have

$$
\left\|\vartheta\left(\nu_{\lambda}\right)-\varpi\right\| \leq\left\|\int_{0}^{6} \mathcal{G}(6, \mathfrak{s})\left[\Psi\left(\mathfrak{s}, v_{\lambda}(\mathfrak{s}), \int_{0}^{\mathfrak{s}} \mathfrak{q}(\mathfrak{s}, \zeta) \varphi(\mathfrak{s}, \zeta, v(\zeta)) d \zeta\right)-\varrho(\mathfrak{s})\right] d \mathfrak{s}\right\| .
$$

By the compactness of operators $\mathcal{K}_{v}(\mathfrak{t})$ and $\mathcal{S}_{v, \mu}(\mathfrak{t})$ for $\mathfrak{t}>0$, one gets the compactness of Green's function $\mathcal{G}(\mathfrak{t}, \mathfrak{s})$ for $\mathfrak{t}, \mathfrak{s}>0$, which implies that

$$
\begin{aligned}
& \left\|\int_{0}^{6} \mathcal{G}(6, \mathfrak{s})\left[\Psi\left(\mathfrak{s}, v_{\lambda}(\mathfrak{s}), \int_{0}^{\mathfrak{s}} \mathfrak{q}(\mathfrak{s}, \zeta) \varphi(\mathfrak{s}, \zeta, v(\zeta)) d \zeta\right)-\varrho(\mathfrak{s})\right] d \mathfrak{s}\right\| \\
& \quad \longrightarrow 0 \quad \text { as } \lambda \longrightarrow 0^{+} .
\end{aligned}
$$


Thus from Eq. (4.7) and Eq. (4.8) we get

$$
\left\|\vartheta\left(v_{\lambda}\right)-\varpi\right\| \longrightarrow 0 \quad \text { as } \lambda \longrightarrow 0^{+}
$$

Equation (4.5) implies that

$$
\begin{aligned}
\left\|v_{\lambda}(6)-v_{b}\right\| & \leq\left\|\lambda \mathcal{R}\left(\lambda, \Gamma_{0}^{6}\right) \vartheta\left(v_{\lambda}\right)\right\| \\
& \leq\left\|\lambda \mathcal{R}\left(\lambda, \Gamma_{0}^{6}\right) \varpi\right\|+\left\|\lambda \mathcal{R}\left(\lambda, \Gamma_{0}^{6}\right)\right\| \cdot\left\|\vartheta\left(v_{\lambda}\right)-\varpi\right\| \\
& \longrightarrow 0 \quad \text { as } \lambda \longrightarrow 0^{+} \text {(by the stated condition and Eq. (4.9)). }
\end{aligned}
$$

Hence the approximate controllability of Eq. (1.1).

\section{Applications}

Here we investigate the proposed results for the following Cauchy problem:

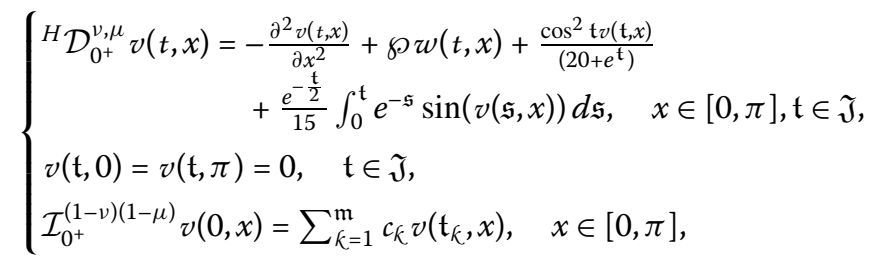

in the Banach space $\mathcal{V}=C^{\eta}[0, \pi], 0<\eta<1$, where $v=\frac{1}{4}, \mu=\frac{1}{2}, \wp>0$ and $c_{\kappa} \in \mathbb{R}$, $\mathcal{K}=1,2, \ldots, \mathfrak{m}$, are such that $\sum_{\kappa=1}^{\mathfrak{m}}\left|c_{k}\right|<\frac{1}{\mathfrak{M}_{S}}$. On substituting $v(t, x)=v(t)$, Eq. (5.1) reduces to Eq. (1.1) with $\mathcal{A}=\frac{-\partial^{2} v}{\partial x^{2}}$ and $D(\mathcal{A})=\left\{v \in C^{\eta+2}[0, \pi]\right.$ such that $\left.v(\mathfrak{t}, 0)=v(\mathfrak{t}, \pi)=0\right\}$. It follows from article [37] that there exist constants $\rho, \epsilon>0$ such that $\mathcal{A}+\rho \in \Theta_{\frac{\pi}{2}-\epsilon}^{\frac{\eta}{2}-1}(\mathcal{V})$. The compactness of semigroup $\{\mathfrak{T}(\mathfrak{t})\}$ follows from (Lemma 4.66) [42]. Since $D(\mathcal{A}) \in \mathcal{C}^{2+\eta}[0, \pi]$ and $C^{2+\eta}[0, \pi]$ is embedded in $C^{\eta}[0, \pi]$, the compactness of resolvent operators follows for every $\eta>0$.

$$
\Psi\left(\mathfrak{t}, v(\mathfrak{t}), \int_{0}^{\mathfrak{t}} \mathfrak{q}(\mathfrak{t}, \mathfrak{s}) \varphi(\mathfrak{t}, \mathfrak{s}, v(\mathfrak{s})) d \mathfrak{s}\right)=\frac{\cos ^{2} \mathfrak{t} v(\mathfrak{t})}{\left(20+e^{\mathfrak{t}}\right)}+\frac{e^{\frac{-\mathfrak{t}}{2}}}{15} \int_{0}^{\mathfrak{t}} e^{-\mathfrak{s}} \sin v(\mathfrak{s}) d \mathfrak{s} .
$$

The bounded linear operator $\Upsilon: \mathcal{W}=\mathcal{V} \longrightarrow \mathcal{V}$ is defined as $\Upsilon w(\mathfrak{t})=\wp w(\mathfrak{t})$.

Following this discussion and the definition of function $\Psi$ and bounded operator $\Upsilon$, it is easy to verify that assumptions (A1)-(A5) hold with

$$
\mathfrak{N}_{1}=\frac{1}{20+e^{6}}, \quad \mathcal{N}_{2}=\frac{e^{\frac{-6}{2}}}{15}, \quad \bar{w}=\left(1-e^{-6}\right), \quad \text { and } \quad \mathfrak{q}^{*}=1
$$

Hence the existence and approximate controllability of Eq. (5.1) follow from Theorem 3.1 and Theorem 4.1 respectively.

\section{Conclusion}

In this paper, we discussed the approximate controllability of Hilfer fractional differential equations with almost sectorial operators. We first prove the existence of mild solutions for similar equations by applying fixed point theory. We will try to investigate the exact controllability and stability of a similar problem in our future research work. 


\section{Acknowledgements}

The first author (Pallavi Bedi) acknowledges the support of Council of Scientific and Industrial Research (CSIR)-New Delhi, India, and the third author (Thabet Abdeljawad) acknowledges the support of Prince Sultan University for funding this work through research group in Applied Mathematics (NAMAM) group number RG-DES-201701-17.

\section{Funding}

Prince Sultan University for funding this work through research group in Applied Mathematics (NAMAM) group number RG-DES-201701-17.

\section{Availability of data and materials}

Not applicable.

\section{Competing interests}

The authors declare that they have no competing interests.

\section{Authors' contributions}

All the authors have made equal contributions in this article. All authors read and approved the final manuscript.

\section{Author details}

'Department of Mathematics and Statistics, Central University of Punjab, Bathinda, 151001 Punjab, India. ${ }^{2}$ Department of Mathematics and General Sciences, Prince Sultan University, 66833, 11586 Riyadh, Saudi Arabia. ${ }^{3}$ Department of Medical Research, China Medical University, 40402, Taichung, Taiwan. ${ }^{4}$ Department of Computer Science and Information Engineering, Asia University, Taichung, Taiwan. ${ }^{5}$ Department of Mathematics, College of Science, Princess Nourah Bint Abdulrahman University, Riyadh, Saudi Arabia.

\section{Publisher's Note}

Springer Nature remains neutral with regard to jurisdictional claims in published maps and institutional affiliations.

Received: 3 July 2020 Accepted: 21 October 2020 Published online: 31 October 2020

\section{References}

1. Alkhazzan, A., Jiang, P., Baleanu, D., Khan, H., Khan, A.: Stability and existence results for a class of nonlinear fractional differential equations with singularity. Math. Methods Appl. Sci. 41(18), 9321-9334 (2018). https://doi.org/10.1002/mma.5263

2. Bedi, P., Kumar, A., Abdeljawad, T., Khan, A.: Existence of mild solutions for impulsive neutral Hilfer fractional evolution equations. Adv. Differ. Equ. 2020, 155 (2020). https://doi.org/10.1186/s13662-020-02615-y

3. Bedi, P., Kumar, A., Abdeljawad, T., Khan, A.: S-asymptotically $\omega$-periodic mild solutions and stability analysis of Hilfer fractional evolution equations. Evol. Equ. Control Theory (2020). https://doi.org/10.3934/eect.2020089

4. Chang, Y.K., Pereira, A., Ponce, R.: Approximate controllability for fractional differential equations of Sobolev type via properties on resolvent operators. Fract. Calc. Appl. Anal. 20(4), 963-987 (2017)

5. Chen, P., Zhang, X.: Approximate controllability of nonlocal problem for non-autonomous stochastic evolution equations. Evol. Equ. Control Theory (2019). https://doi.org/10.3934/eect.2020076

6. Chen, P., Zhang, X., Li, Y.: Approximation technique for fractional evolution equations with nonlocal integral conditions. Mediterr. J. Math. 14(6), 226 (2017). https://doi.org/10.1007/s00009-017-1029-0

7. Chen, P., Zhang, X., Li, Y.: A blowup alternative result for fractional nonautonomous evolution equation of Volterra type. Commun. Pure Appl. Anal. 17(5), 1975-1992 (2018)

8. Chen, P., Zhang, X., Li, Y.: Non-autonomous parabolic evolution equations with non-instantaneous impulses governed by noncompact evolution families. J. Fixed Point Theory Appl. 21(3), 84 (2019). https://doi.org/10.1007/s11784-019-0719-6

9. Chen, P., Zhang, X., Li, Y.: Non-autonomous evolution equations of parabolic type with non-instantaneous impulses. Mediterr. J. Math. 16(5), 118 (2019). https://doi.org/10.1007/s00009-019-1384-0

10. Chen, P., Zhang, X., Li, Y.: Fractional non-autonomous evolution equation with nonlocal conditions. J. Pseudo-Differ. Oper. Appl. 10(4), 955-973 (2019). https://doi.org/10.1007/s11868-018-0257-9

11. Chen, P., Zhang, X., Li, Y.: Existence and approximate controllability of fractional evolution equations with nonlocal conditions via resolvent operators. Fract. Calc. Appl. Anal. 23(1), 268-291 (2020). https://doi.org/10.1515/fca-2020-0011

12. Chen, P., Zhang, X., Li, Y.: Approximate controllability of non-autonomous evolution system with nonlocal conditions. J. Dyn. Control Syst. 26(1), 1-16 (2020). https://doi.org/10.1007/s10883-018-9423-x

13. Chen, P., Zhang, X., Li, Y.: Cauchy problem for fractional non-autonomous evolution equations. Banach J. Math. Anal. 14(2), 559-584 (2020). https://doi.org/10.1007/s43037-019-00008-2

14. Debbouche, A., Antonov, V.: Approximate controllability of semilinear Hilfer fractional differential inclusions with impulsive control inclusion conditions in Banach spaces. Chaos Solitons Fractals 102, 140-148 (2017). https://doi.org/10.1016/j.chaos.2017.03.023

15. Devi, A., Kumar, A., Abdeljawad, T., Khan, A.: Existence and stability analysis of solutions for fractional Langevin equation with nonlocal integral and anti-periodic type boundary conditions. Fractals (2020). https://doi.org/10.1142/S0218348X2040006X

16. Ding, X.L., Ahmad, B.: Analytical solutions to fractional evolution equations with almost sectorial operators. Adv. Differ Equ. 2016(1), 203 (2016). https://doi.org/10.1186/s13662-016-0927-y

17. Du, J., Jiang, W., Niazi, A.U.K.: Approximate controllability of impulsive Hilfer fractional differential inclusions. J. Nonlinear Sci. Appl. 10(2), 595-611 (2017) 
18. Fu, X.: Approximate controllability of semilinear non-autonomous evolution systems with state-dependent delay. Evol. Equ. Control Theory 6(4), 517-534 (2017). https://doi.org/10.3934/eect.2017026

19. Gomez-Aguilar, J.F., Cordova-Fraga, T., Abdeljawad, T., Khan, A., Khan, H.: Analysis of fractal-fractional malaria transmission model. Fractals (2020). https://doi.org/10.1142/S0218348X20400411

20. Gu, H., Trujillo, J.J.: Existence of mild solution for evolution equation with Hilfer fractional derivative. Appl. Math. Comput. 257, 344-354 (2015). https://doi.org/10.1016/j.amc.2014.10.083

21. Jaiswal, A., Bahuguna, D.: Hilfer fractional differential equations with almost sectorial operators. Differ. Equ. Dyn. Syst. (2020). https://doi.org/10.1007/s12591-020-00514-y

22. Khan, A., Khan, T.S., Syam, M.I., Khan, H.: Analytical solutions of time-fractional wave equation by double Laplace transform method. Eur. Phys. J. Plus 134(4), 163 (2019). https://doi.org/10.1140/epjp/i2019-12499-y

23. Khan, A., Shah, K., Li, Y., Khan, T.S.: Ulam type stability for a coupled system of boundary value problems of nonlinear fractional differential equations. J. Funct. Spaces (2017). https://doi.org/10.1155/2017/3046013

24. Khan, A., Syam, M.I., Zada, A., Khan, H.: Stability analysis of nonlinear fractional differential equations with Caputo and Riemann-Liouville derivatives. Eur. Phys. J. Plus 133(7), 264 (2018). https://doi.org/10.1140/epjp/i2018-12119-6

25. Khan, H., Alipour, M., Khan, R.A., Tajadodi, H., Khan, A.: On approximate solution of fractional order logistic equations by operational matrices of Bernstein polynomials. J. Math. Comput. Sci. 14, 222-232 (2015)

26. Khan, H., Chen, W., Khan, A., Khan, T.S., Al-Madlal, Q.M.: Hyers-Ulam stability and existence criteria for coupled fractional differential equations involving p-Laplacian operator. Adv. Differ. Equ. 2018(1), 455 (2018) https://doi.org/10.1186/s13662-018-1899-x

27. Khan, H., Gomez Aguilar, J.F., Abdeljawad, T., Khan, A.: Existence results and stability criteria for ABC-fuzzy-Volterra integro-differential equation. Fractals (2020). https://doi.org/10.1142/S0218348X20400484

28. Khan, H., Gómez-Aguilar, J.F., Alkhazzan, A., Khan, A.: A fractional order HIV-TB coinfection model with nonsingular Mittag-Leffler law. Math. Methods Appl. Sci. 43(6), 3786-3806 (2020). https://doi.org/10.1002/mma.6155

29. Khan, H., Tunç, C., Alkhazan, A., Ameen, B., Khan, A.: A generalization of Minkowski's inequality by Hahn integral operator. J. Taibah Univ. Sci. 12(5), 506-513 (2018). https://doi.org/10.1080/16583655.2018.1493859

30. Khan, Z.A.: Integral inequality of Gronwall type with an application. J. Math. Comput. Sci. 5(1), 34-41 (2015)

31. Khan, Z.A.: Solvability for a class of integral inequalities with maxima on the theory of time scales and their applications. Bound. Value Probl. 2019, 146 (2019). https://doi.org/10.1186/s13661-019-1259-0

32. Khan, Z.A.: Analysis on some powered integral inequalities with retarded argument and application. J. Taibah Univ. Sci. 14(1), 488-495 (2020). https://doi.org/10.1080/16583655.2020.1747218

33. Li, F.: Mild solutions for abstract fractional differential equations with almost sectorial operators and infinite delay. Adv. Differ. Equ. 2013(1), 327 (2013). https://doi.org/10.1186/1687-1847-2013-327

34. LV, J., Yang, X.: Approximate controllability of Hilfer fractional neutral stochastic differential equations. Dyn. Syst. Appl. 27(4), 691-713 (2018). https://doi.org/10.12732/dsa.v27i4.1

35. LV , J., Yang, X.: Approximate controllability of Hilfer fractional differential equations. Math. Methods Appl. Sci. 43(1), 242-254 (2020). https://doi.org/10.1002/mma.5862

36. Mahmudov, N.I., McKibben, M.A.: On the approximate controllability of fractional evolution equations with generalized Riemann-Liouville fractional derivative. J. Funct. Spaces 2015, Article ID 263823 (2015) https://doi.org/10.1155/2015/263823

37. Periago, F., Straub, B.: A functional calculus for almost sectorial operators and applications to abstract evolution equations. J. Evol. Equ. 2(1), 41-68 (2002). https://doi.org/10.1007/s00028-002-8079-9

38. Podlubny, I.: Fractional Differential Equations. Academic Press, San Diego (1999)

39. Ullah, M., Sarwar, M., Khan, H., Abdeljawad, T., Khan, A.: Near-coincidence point results in metric interval space and hyperspace via simulation functions. Adv. Differ. Equ. 2020(1), 291 (2020). https://doi.org/10.1186/s13662-020-02734-6

40. Wang, J., Fečkan, M., Zhou, Y.: Approximate controllability of Sobolev type fractional evolution systems with nonlocal conditions. Evol. Equ. Control Theory 6(3), 471 (2017)

41. Yang, M., Wang, Q.R.: Approximate controllability of Hilfer fractional differential inclusions with nonlocal conditions. Math. Methods Appl. Sci. 40(4), 1126-1138 (2017). https://doi.org/10.1002/mma.4040

42. Yong, Z., Jinrong, W., Lu, Z.: Basic Theory of Fractional Differential Equations. World Scientific, Singapore (2016)

43. Zhang, L., Zhou, Y.: Fractional Cauchy problems with almost sectorial operators. Appl. Math. Comput. 257, 145-157 (2015). https://doi.org/10.1016/j.amc.2014.07.024

44. Zhou, Y., Vijayakumar, V., Ravichandran, C., Murugesu, R.: Controllability results for fractional order neutral functional differential inclusions with infinite delay. Fixed Point Theory 18(2), 773-798 (2017).

https://doi.org/10.24193/fpt-ro.2017.2.62 